\begin{tabular}{|c|l|}
\hline Title & Effect of magnetic ani sotropy on sky rmions with a high topological number in itinerant magnets \\
\hline Author(s) & Hayami, Satoru; Motome, Y ukitoshi \\
\hline Citation & $\begin{array}{l}\text { Physical review B, 99(9), 094420 } \\
\text { https://doi.org/10.1103/PhysRevB.99.094420 }\end{array}$ \\
\hline Issue Date & 2019-03-18 \\
\hline Doc URL & http://hdl.handle.net/2115/75583 \\
\hline Rights & ○2019A A merican Physical Society \\
\hline Type & article \\
\hline File Information & PhysRevB.99.094420.pdf \\
\hline
\end{tabular}

Instructions for use 


\title{
Effect of magnetic anisotropy on skyrmions with a high topological number in itinerant magnets
}

\author{
Satoru Hayami ${ }^{1}$ and Yukitoshi Motome ${ }^{2}$ \\ ${ }^{1}$ Faculty of Science, Hokkaido University, Sapporo 060-0810, Japan \\ ${ }^{2}$ Department of Applied Physics, University of Tokyo, Tokyo 113-8656, Japan
}

(Received 21 November 2018; published 18 March 2019)

\begin{abstract}
We report our numerical results for the effect of magnetic anisotropy on a skyrmion crystal with a high topological number of two, which was recently discovered in an itinerant electron model [R. Ozawa, S. Hayami, and Y. Motome, Phys. Rev. Lett. 118, 147205 (2017)]. By performing numerical simulations based on the kernel polynomial method and the Langevin dynamics for the Kondo lattice model on a triangular lattice, we find that the topological property remains robust against single-ion anisotropy, while the magnetic texture is deformed continuously. The resultant spin structure is characterized by three wave numbers (triple- $Q$ state), in which the $x y$ component of the spins forms a magnetic vortex crystal and the $z$ component of the spins behaves as a sinusoidal wave. For a larger anisotropy, we show that the system exhibits a phase transition from a skyrmion crystal to topologically trivial phases with vanishing scalar chirality: a single- $Q$ collinear state and double$Q$ noncoplanar states for the easy-axis and easy-plane anisotropy, respectively. We also examine the effect of single-ion anisotropy in an external magnetic field, and find that the field range of the skyrmion crystal is rather insensitive to the anisotropy, in contrast to another skyrmion crystal with a topological number of one whose field range is considerably extended (reduced) by the easy-axis (easy-plane) anisotropy.
\end{abstract}

DOI: 10.1103/PhysRevB.99.094420

\section{INTRODUCTION}

Spin scalar chirality, which is defined by a triple scalar product of three spins as $\mathbf{S}_{i} \cdot\left(\mathbf{S}_{j} \times \mathbf{S}_{k}\right)$, has attracted much interest in condensed matter physics. It generates an emergent electromagnetic field for electrons through the spin Berry phase mechanism, which has great potential for the control of electronic states and transport phenomena, such as the anomalous Hall effect called the topological Hall effect [1,2]. In particular, when the scalar chirality acquires a net component by a periodic noncoplanar spin texture, the coherent Berry phase can lead to quantized topological Hall effects [3-6].

Skyrmion crystals (SkXs) are one of the most attracting examples to exhibit the topological Hall effect [7-12]. They are given by a periodic arrangement of swirling spin textures called skyrmions, and are often found in magnets with chiral lattice structures. Such SkXs are stabilized in a magnetic field under competition between the ferromagnetic exchange and the Dzyaloshinskii-Moriya interactions $[13,14]$, the latter of which originates from relativistic spin-orbit coupling under inversion symmetry breaking [15-17]. Another mechanism for stabilizing SkXs has been investigated in frustrated magnets with competing nearest-neighbor ferromagnetic and further-neighbor antiferromagnetic exchange interactions [18-23]. These SkXs show a different feature for magnetic anisotropy: The former SkXs become more robust by introducing both easy-axis and easy-plane anisotropy [24-28], while the latter SkXs are stabilized (destabilized) by easy-axis (plane) anisotropy [19-21,29].

Yet another mechanism for SkXs has been developed mainly from the theoretical side, by fully taking into account the itinerant nature of electrons. The argument is based on Kondo-type exchange coupling between the itinerant electrons and localized spins, which gives rise to effective multiple-spin interactions between localized spins [30-34] in addition to the conventional Ruderman-Kittel-Kasuya-Yosida (RKKY) interaction [35-37]. Such effective interactions result in noncoplanar spin textures on a variety of lattice geometries: triangular [4,5,38-43], honeycomb [42,44], kagome [45,46], square [47-49], cubic [50], face-centered-cubic [6], pyrochlore [51], and Shastry-Sutherland lattices [52]. This mechanism has two interesting features: (i) It does not necessarily require either inversion symmetry breaking or spin-orbit coupling, and (ii) it can produce unconventional SkXs that have not been seen in other mechanisms. For the latter, for instance, a recent theoretical study on the triangular lattice revealed a SkX with a topological number of two $\left(n_{\mathrm{sk}}=2\right)$ at zero magnetic field, and phase transitions with successive changes of the topological number $n_{\text {sk }}=2 \rightarrow 1 \rightarrow 0$ while increasing an external magnetic field [53]. However, such a SkX with $n_{\mathrm{sk}}=2$ has yet to be identified in experiments. Toward experimental observations, it is desirable to examine how it responds to perturbations, such as magnetic anisotropy. It will also be helpful for clarifying the similarity and difference between conventional skyrmions and those rooted in itinerant electrons.

In the present paper, we investigate the effect of single-ion anisotropy on SkXs with $n_{\mathrm{sk}}=2$ and 1 in itinerant magnets. We examine how the spin structures are modulated and how these topological phases are robust against introducing easy-axis or easy-plane anisotropy. By performing large-scale Langevin dynamics simulations enabled by the kernel polynomial method (KPM-LD) [54] for the Kondo lattice model on a triangular lattice, we find that the spin structure of the $n_{\mathrm{sk}}=2 \mathrm{SkX}$ is deformed into an anisotropic form composed of magnetic vortices in the $x y$-spin component and a 
sinusoidal wave in the $z$-spin component. In addition, we show that the SkX shows a topological trivial-nontrivial transition to a single- $Q(1 Q)$ collinear [double- $Q(2 Q)$ noncoplanar] state while increasing the easy-axis (easy-plane) anisotropy. We also compare the robustness of the SkXs with $n_{\mathrm{sk}}=2$ and 1 in an applied magnetic field. We find that the field range of the $n_{\mathrm{sk}}=2$ state is rather insensitive to the anisotropy, while the $n_{\mathrm{sk}}=1$ state is substantially stabilized (destabilized) by the easy-axis (easy-plane) anisotropy similar to conventional SkXs found in other systems.

The rest of the paper is organized as follows. In Sec. II, we introduce the Kondo lattice model including single-ion anisotropy and Zeeman coupling to an external magnetic field, outline the KPM-LD method, and define the observables that we evaluate. We examine the effect of single-ion anisotropy on the $n_{\mathrm{sk}}=2 \mathrm{SkX}$ at zero magnetic field and for a nonzero field in Sec. III. Section IV is devoted to a summary. In Appendix, we present snapshots of the spin configuration in the $n_{\mathrm{sk}}=2 \mathrm{SkX}$ for several values of single-ion anisotropy.

\section{MODEL AND METHOD}

\section{A. Kondo lattice model}

We consider the Kondo lattice model including the effect of single-ion anisotropy and external magnetic field on the triangular lattice. The Hamiltonian is given by

$$
\mathcal{H}=-\sum_{i, j, \sigma} t_{i j} c_{i \sigma}^{\dagger} c_{j \sigma}+J \sum_{i} \mathbf{s}_{i} \cdot \mathbf{S}_{i}-A \sum_{i}\left(S_{i}^{z}\right)^{2}-H \sum_{i} S_{i}^{z}
$$

The first term represents the kinetic energy of itinerant electrons, where $c_{i \sigma}^{\dagger}\left(c_{i \sigma}\right)$ is a creation (annihilation) operator of an itinerant electron at site $i$ and spin $\sigma$. The second term in Eq. (1) represents the on-site exchange coupling between itinerant electron spins $\mathbf{s}_{i}=(1 / 2) \sum_{\sigma, \sigma^{\prime}} c_{i \sigma}^{\dagger} \boldsymbol{\sigma}_{\sigma \sigma^{\prime}} c_{i \sigma^{\prime}}$ and localized spins $\mathbf{S}_{i}$ with a coupling constant $J$, where $\sigma=\left(\sigma^{x}, \sigma^{y}, \sigma^{z}\right)$ is the vector of Pauli matrices. We regard $\mathbf{S}_{i}$ as a classical spin with fixed length $\left|\mathbf{S}_{i}\right|=1$ (the sign of $J$ is irrelevant). The third and fourth terms describe the easy-axis $(A>0)$ or easy-plane $(A<0)$ anisotropy and the Zeeman coupling to an external magnetic field along the $z$ direction, respectively, both of which are taken into account only for the localized spins for simplicity.

The ground state of the model in Eq. (1) was investigated in the absence of single-ion anisotropy $(A=0)$ [53]. The system exhibits a triple- $Q(3 Q) \mathrm{SkX}$ with $n_{\mathrm{sk}}=2$ at zero field, which is characterized by three wave numbers $\mathbf{Q}_{\eta}=$ $R[2 \pi(\eta-1) / 3](\pi / 3,0) \quad(\eta=1-3)$, where $R(\theta)$ represents the rotational operation around the $z$ axis by $\theta$. Furthermore, while increasing the magnetic field, topological phase transitions occur successively as $n_{\mathrm{sk}}=2 \rightarrow 1 \rightarrow 0$. In the following calculations, we examine the effect of single-ion anisotropy on SkXs with $n_{\mathrm{sk}}=2$ and 1 by introducing the third term in Eq. (1) and setting the other parameters at the same values as those in Ref. [53]: the nearest-neighbor and third-neighbor hoppings, $t_{1}=1$ and $t_{3}=-0.85$, respectively, $J=1$, and the chemical potential $\mu=-3.5$.

\section{B. Simulation method}

We investigate the ground state of the Kondo lattice model in Eq. (1) by performing the KPM-LD simulation, which is an unbiased numerical simulation based on Langevin dynamics combined with the kernel polynomial method [54,55]. This method enables calculations for large system sizes, typically up to $10^{4}$ sites, and has been applied to similar models with itinerant electrons [32,34,45,53,54,56-58]. Our simulation is done at zero temperature from initial states with random spin configurations for a $96^{2}$-site cluster of the triangular lattice with periodic boundary conditions in both directions. In the kernel polynomial method, we expand the density of states by up to 2000th order of the Chebyshev polynomials with $16^{2}$ random vectors [59]. In the Langevin dynamics, we use a projected Heun scheme [60] for 1000-5000 steps with the time interval $\Delta \tau=2$.

\section{Physical observables}

For spin configurations obtained by the KPM-LD simulation, we calculate the spin structure factor to identify each magnetic phase, which is given by

$$
S(\mathbf{q})=S^{x x}(\mathbf{q})+S^{y y}(\mathbf{q})+S^{z z}(\mathbf{q}),
$$

where

$$
S^{\alpha \alpha}(\mathbf{q})=\frac{1}{N} \sum_{j, l} S_{j}^{\alpha} S_{l}^{\alpha} e^{i \mathbf{q} \cdot\left(\mathbf{r}_{j}-\mathbf{r}_{l}\right)}
$$

with $\alpha=x, y, z$ and $N$ is the system size. We also compute

$$
S^{\perp}(\mathbf{q})=S^{x x}(\mathbf{q})+S^{y y}(\mathbf{q}) .
$$

In addition, we introduce the following notation for the magnetic moments with wave number $\mathbf{q}$,

$$
m_{\mathbf{q}}=\sqrt{\frac{S(\mathbf{q})}{N}} .
$$

Note that the uniform magnetization is given by $M=m_{\mathbf{q}=\mathbf{0}}$. In addition, in order to identify whether or not the obtained phase is chiral, we calculate the net spin scalar chirality of the localized spins, which is defined as

$$
\chi_{\mathrm{sc}}=\frac{1}{N} \sum_{p} \chi_{p}=\frac{1}{N} \sum_{p} \mathbf{S}_{i} \cdot\left(\mathbf{S}_{j} \times \mathbf{S}_{k}\right)
$$

where $\chi_{p}$ is a local scalar chirality defined on the triangle plaquette $p ; i, j, k$ are sites on each triangle plaquette $p$ in the counterclockwise direction. The scalar chirality $\chi_{p}$ is related with the topological number $n_{\text {sk }}$ as

$$
n_{\mathrm{sk}}=\frac{1}{2 \pi} \sum_{p \in \text { u.c. }} \frac{\chi_{p}}{\left(\mathbf{S}_{i}+\mathbf{S}_{j}+\mathbf{S}_{k}\right)^{2}-1},
$$

where the summation $p$ is taken for the magnetic unit cell (u.c.).

\section{RESULTS}

In this section, we present numerical results for the effect of single-ion anisotropy obtained by the KPM-LD method. In Sec. III A, we show how the SkX with $n_{\text {sk }}=2$ is affected by the anisotropy at zero field. We extend the study to nonzero 


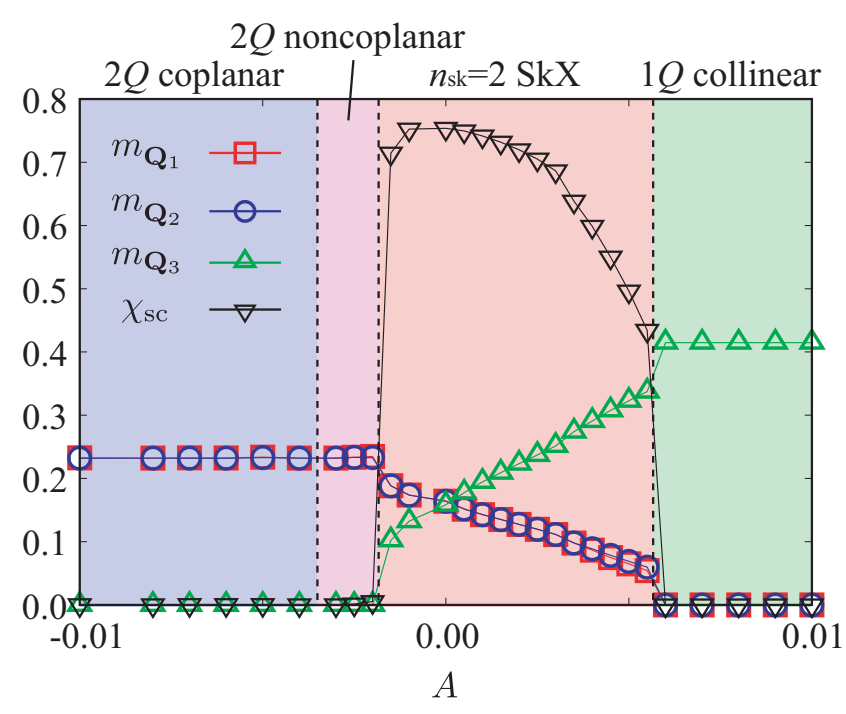

FIG. 1. KPM-LD results for the model in Eq. (1) at zero field: single-ion anisotropy $A$ dependences of the $\mathbf{Q}_{v}$ components of the magnetization [Eq. (5)] and the net spin scalar chirality [Eq. (6)]. The vertical dashed lines show the phase boundaries.

fields and compare the robustness of SkXs with $n_{\mathrm{sk}}=2$ and $n_{\mathrm{sk}}=1$ in Sec. III B.

\section{A. At zero field}

First, we present the KPM-LD results at zero field $H=0$. Figure 1 shows the $\mathbf{Q}_{v}$ component of the magnetization $m_{\mathbf{Q}_{v}}$ [Eq. (5)] and the net spin scalar chirality $\chi_{\text {sc }}$ [Eq. (6)] as functions of single-ion anisotropy $A$. At $A=0$, the $3 Q \mathrm{SkX}$ with $n_{\text {sk }}=2$ is realized as discussed in Ref. [53]. In this state, the amplitudes of the $\mathbf{Q}_{1}, \mathbf{Q}_{2}$, and $\mathbf{Q}_{3}$ components are equivalent, i.e., $m_{\mathbf{Q}_{1}}=m_{\mathbf{Q}_{2}}=m_{\mathbf{Q}_{3}}$, as shown in Fig. 1. At the same time, the noncoplanar spin structure leads to the nonzero value of $\chi_{\mathrm{sc}}$; the resultant topological number is quantized at two [53]. Note that the helicity of the $n_{\mathrm{sk}}=2 \mathrm{SkX}$ at $A=H=0$ is arbitrary because of spin rotational symmetry.

When single-ion anisotropy is introduced $(A \neq 0)$, the $x y$ and $z$ components of the magnetization behave differently due to the breaking of rotational symmetry in spin space. The $x y$ component shows the $2 Q$ structures with equal intensities at $\mathbf{Q}_{1}$ and $\mathbf{Q}_{2}$, while the $z$ component shows the $1 Q$ structure at $\mathbf{Q}_{3}$, as shown in Fig. 1. We show the snapshots of the spin configuration in Fig. 2(a). (The choice of $\mathbf{Q}_{v}$ for $2 Q$ and $1 Q$ depends on the snapshot.) The intensities of the $2 Q$ peaks in the $x y$ component are smaller (larger) than that of the $1 Q$ peak in the $z$ component for the easy-axis anisotropy $A>0$ (easy-plane anisotropy $A<0$ ). We also present the spin configurations for several values of $A$ in Appendix. We confirm that the topological number in Eq. (7) remains unchanged at $n_{\mathrm{sk}}=2$ for the continuous modulations of the spin structures by $A$. From these observations, we find that the real-space spin configuration of the $3 Q \mathrm{SkX}$ with $n_{\mathrm{sk}}=2$ is approximately given by

$$
\mathbf{S}_{i} \propto\left[\cos \left(\mathbf{Q}_{1} \cdot \mathbf{r}_{i}\right), \cos \left(\mathbf{Q}_{2} \cdot \mathbf{r}_{i}\right), a^{z} \cos \left(\mathbf{Q}_{3} \cdot \mathbf{r}_{i}\right)\right],
$$

where the coefficient $a^{z}$ depends on $A: a^{z}>1\left(a^{z}<1\right)$ for $A>0(A<0)$. Note that the $x y$ component in Eq. (8) is generally described by an arbitrary linear combination of $S_{i}^{x}$ and $S_{i}^{y}$ owing to the rotational symmetry around the $z$ axis. Thus, the spin texture is modified by single-ion anisotropy into a superposition of the $2 Q$ vortices in the $x y$ component and a sinusoidal wave in the $z$ component. The helicity for the $2 Q$ vortices is arbitrary, which reflects the presence of rotational symmetry in the $x y$ component of spins. Interestingly, the

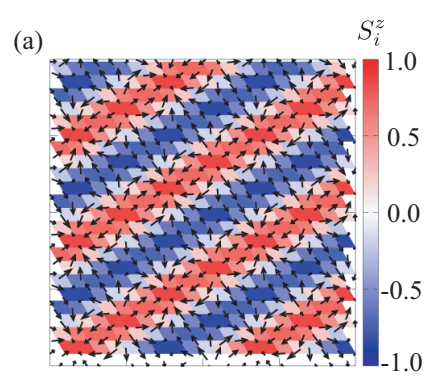

(b)

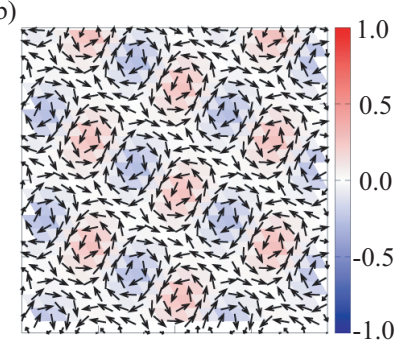

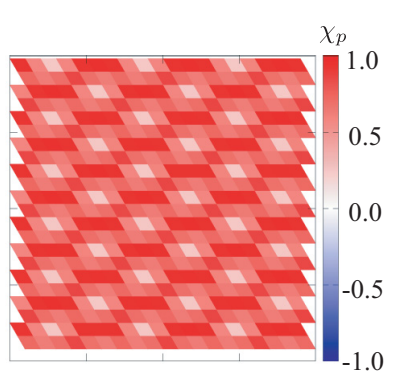

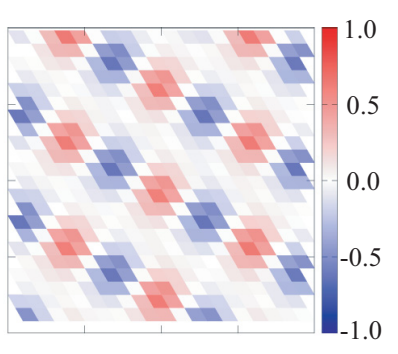

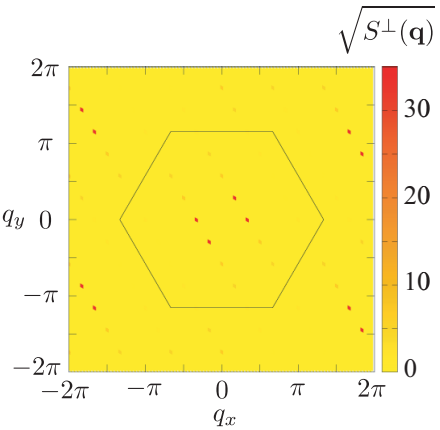

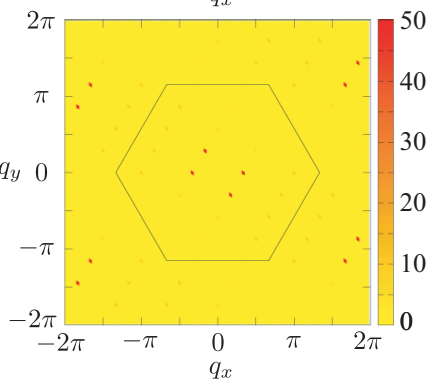

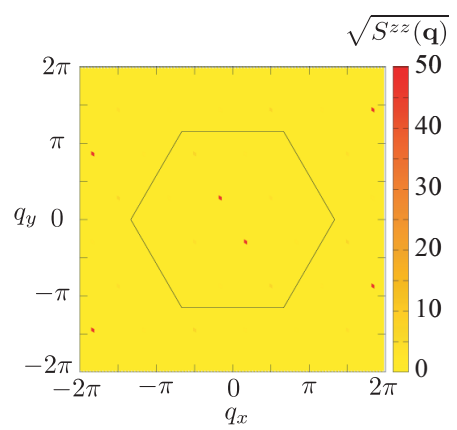

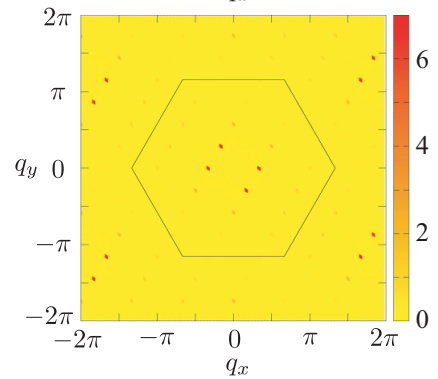

FIG. 2. Leftmost: Snapshots of the spin configurations in (a) the $3 Q$ SkX with $n_{\mathrm{sk}}=2$ for $A=0.0025$ and (b) the $2 Q$ noncoplanar state for $A=-0.0025$ at $H=0$. The contour shows the $z$ component of the spin moment. Middle left: Snapshots of the spin scalar chirality. Middle right and rightmost: The square root of the $x y$ and $z$ components of the spin structure factor, respectively. In the right two columns, the hexagons represent the first Brillouin zone. 

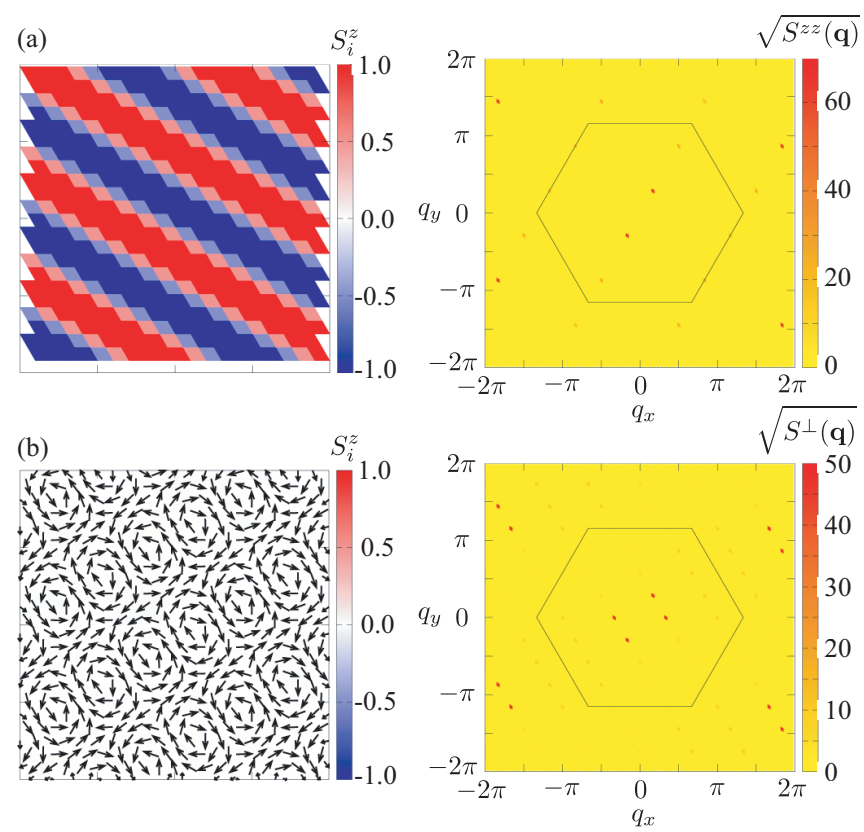

FIG. 3. Left: Snapshots of the spin configurations in (a) the $1 Q$ collinear state for $A=0.008$ and (b) the $2 Q$ coplanar state for $A=$ -0.006 at $H=0$. The contour shows the $z$ component of the spin moment. Right: The square root of the $z(x y)$ component of the spin structure factor in the upper panel (lower). The $x y(z)$ component of the spin structure factor is negligible in (a) [(b)]. In the right column, the hexagons represent the first Brillouin zone.

real-space spin structures lack clear skyrmion cores, in stark contrast to conventional SkXs, as shown in Fig. 2(a).

While increasing the easy-axis anisotropy, the $z$ component is developed almost linearly to $A$, while the $x$ and $y$ components are suppressed, as shown in Fig. 1. Simultaneously, the net scalar chirality decreases monotonically by increasing $A$, which indicates that the solid angle spanned by three spins becomes smaller for larger $A$. At $A \sim 0.006$, the SkX with $n_{\mathrm{sk}}=2$ turns into a $1 Q$ collinear state with a sudden change of the topological number from $n_{\mathrm{sk}}=2$ to 0 . This is a first-order transition with vanishing spin scalar chirality $\chi_{\text {sc }}$ [see Eq. (7)], as shown in Fig. 1. In this $1 Q$ collinear phase, the $z$ component of the spin structure factor shows a $1 Q$ peak as shown in Fig. 3(a), while the $x y$ component is negligibly small.

Meanwhile, for the easy-plane anisotropy $A<0$, the $n_{\mathrm{sk}}=$ 2 SkX turns into a $2 Q$ noncoplanar state with $n_{\mathrm{sk}}=0$ at $A \sim-0.002$, as shown in Fig. 1 . In this case also, $\chi_{\text {sc }}$ suddenly vanishes due to the topological transition. The typical spin configuration for the $2 Q$ noncoplanar state is shown in Fig. 2(b). In this state, the spin configuration is characterized by the $2 Q$ modulation: Both the $x y$ and $z$ components of the spin structure factor have two dominant peaks, while the intensities of the $x y$ component are much larger than that of the $z$ component in Fig. 2(b). The net scalar chirality is zero in this phase, although there is a $2 Q$ chiral density wave [see the middle left panel of Fig. 2(b)]. While further increasing the easy-plane anisotropy, the small $z$ component of the magnetization is suppressed to zero and the system turns into a $2 Q$ coplanar state at $A \sim-0.0035$, whose spin structure is shown in Fig. 3(b).
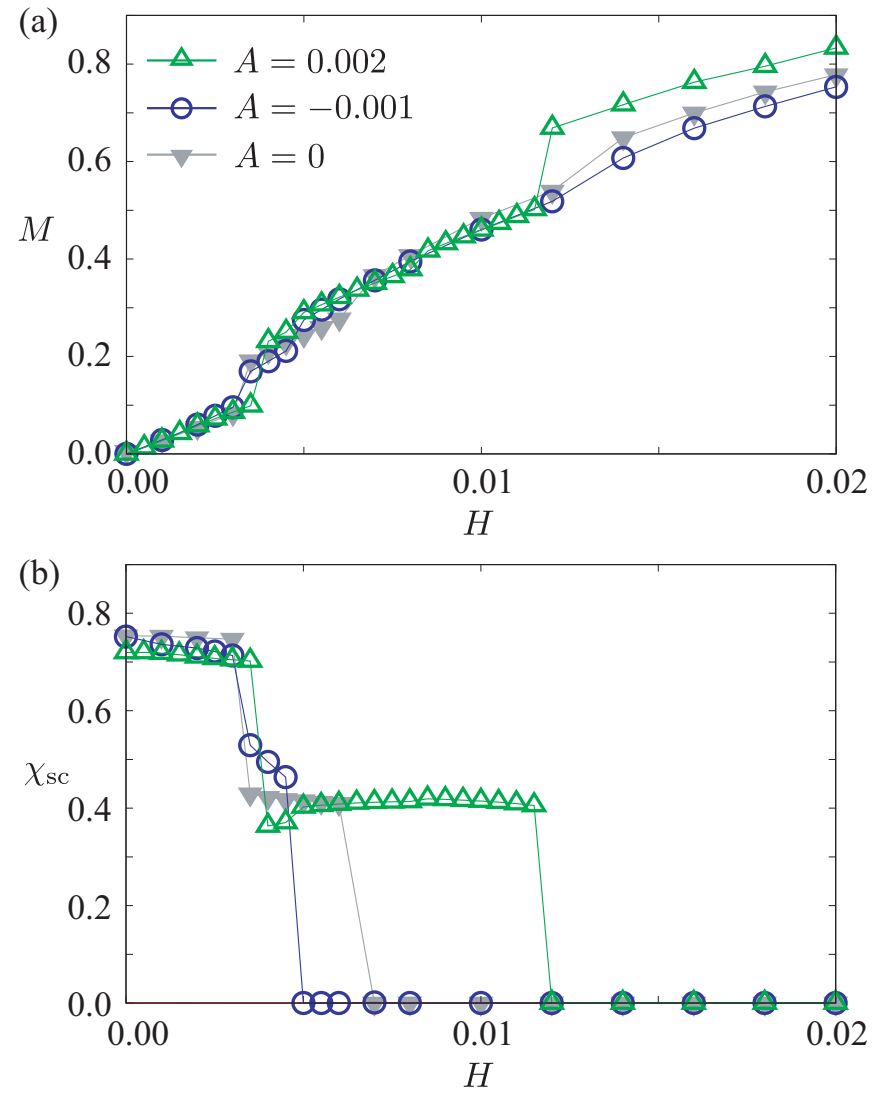

(c)

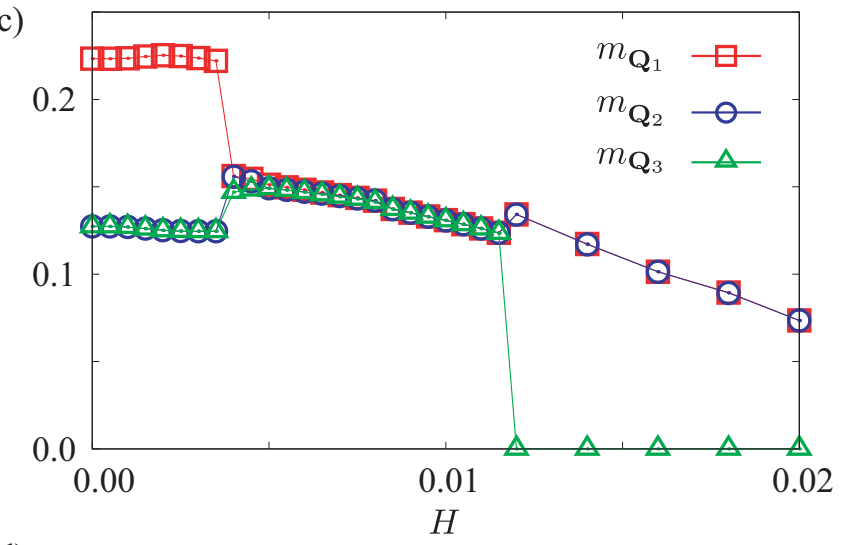

(d) 0.2

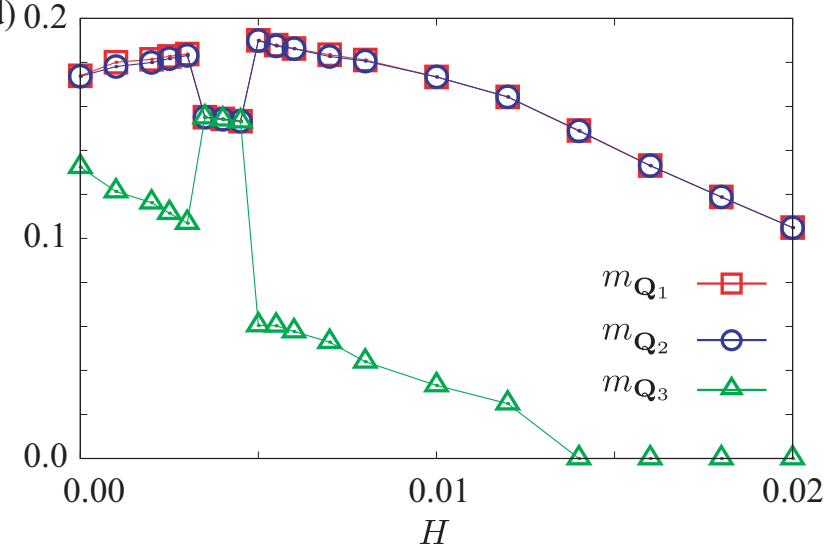

FIG. 4. (a) Magnetization curves and (b) the net scalar chirality for $A=0.002$ and -0.001 under the magnetic field $H$. The data at $A=0$ are also shown for comparison. (c), (d) $H$ dependences of $m_{\mathbf{Q}_{v}}$ ( $v=1-3$ ) for (c) $A=0.002$ and (d) $A=-0.001$. 

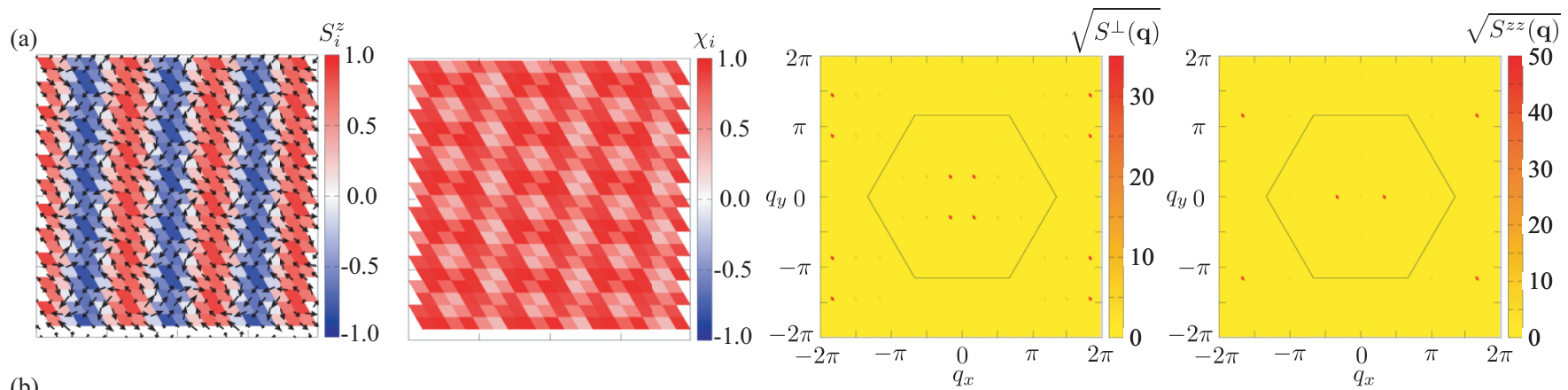

(b)
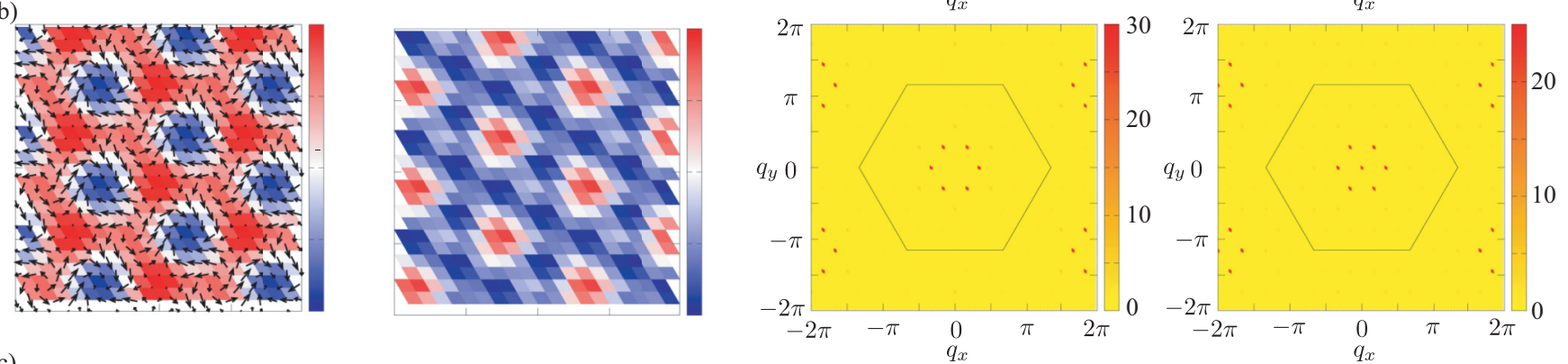

(c)
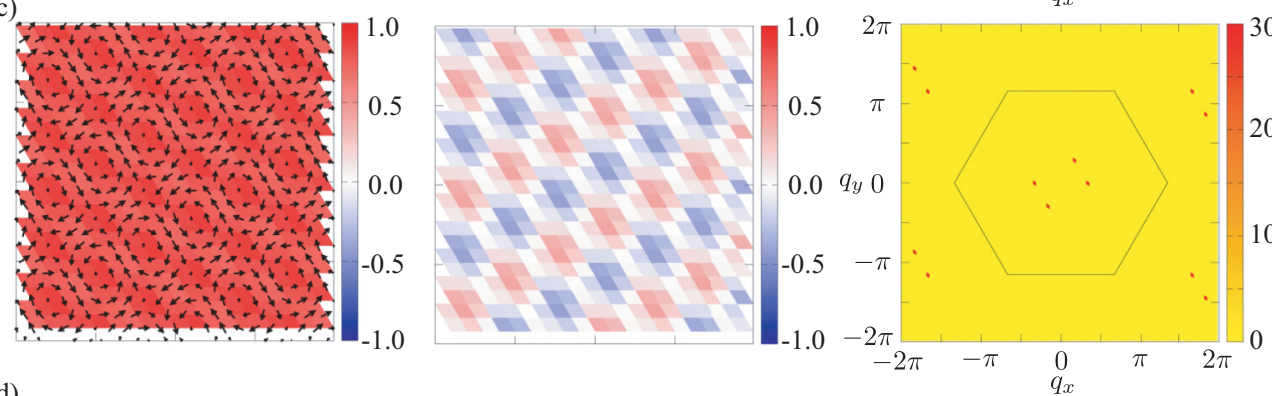

(d)
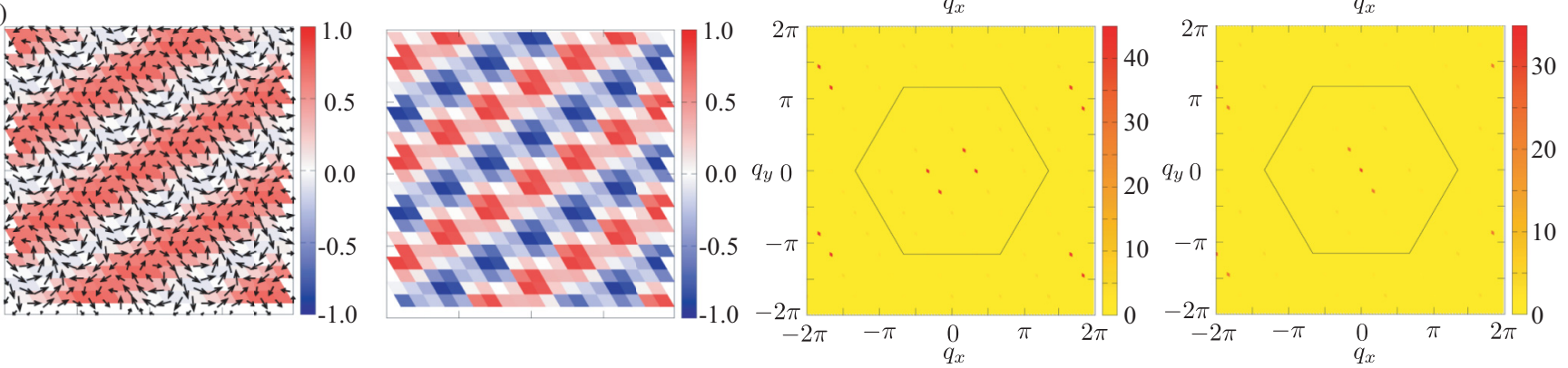

FIG. 5. Leftmost: Snapshots of the spin configurations in (a) the $3 Q \mathrm{SkX}$ with $n_{\mathrm{sk}}=2$ for $H=0.002$ and $A=0.002$, (b) the $3 Q$ SkX with $n_{\text {sk }}=1$ for $H=0.004$ and $A=0.002$, (c) the $2 Q$ state with $n_{\text {sk }}=0$ for $H=0.018$ and $A=0.002$, and (d) the $3 Q$ state with $n_{\text {sk }}=0$ for $H=0.006$ and $A=-0.001$. The contour shows the $z$ component of the spin moment. Middle left: Snapshots of the spin scalar chirality. Middle right and rightmost: The square root of the $x y$ and $z$ components of the spin structure factor, respectively. In the right two columns, the hexagons represent the first Brillouin zone. In (c), the field-induced $\mathbf{q}=\mathbf{0}$ component is subtracted for clarity.

\section{B. In a magnetic field}

Next, we discuss the effect of the magnetic field on the $n_{\mathrm{sk}}=2 \mathrm{SkX}$ in the presence of single-ion anisotropy $A$. In the previous study at $A=0$, while increasing the magnetic field $H$, the $3 Q \mathrm{SkX}$ with $n_{\mathrm{sk}}=2$ changes to another $3 Q$ $\mathrm{SkX}$ with $n_{\mathrm{sk}}=1$, and finally turns into a topologically trivial $3 Q$ noncoplanar state with $n_{\mathrm{sk}}=0$ [53]. We here compute the magnetic states at $A \neq 0$ and $H \neq 0$, and examine how the $n_{\mathrm{sk}}=2 \mathrm{SkX}$ is robust against $H$ in comparison with the $n_{\mathrm{sk}}=1 \mathrm{SkX}$.

Figure 4(a) shows the magnetization curves at $A=0.002$ and $A=-0.001$ obtained by the KPM-LD simulations. We plot the result at $A=0$ for comparison, which reproduces the previous result [53]. In the low-field region, the magnetizations continuously increase with $H$, and the spin structures for $A \neq 0$ are similar to those at $A=0$, as exemplified in Fig. 5(a). While further increasing $H$, the magnetizations show a jump at $H \sim 0.003$ almost irrespective of $A$. This is the topological phase transition from the $3 Q \mathrm{SkX}$ with $n_{\mathrm{sk}}=2$ to another $3 Q \mathrm{SkX}$ with $n_{\mathrm{sk}}=1$. In the field-induced SkXs, the cores of swirling spin textures with $S_{i}^{z}=-1$ form a triangular lattice, as shown in the snapshot in Fig. 5(b). In this state, the magnetization has equal weights for the $\mathbf{Q}_{v}$ components ( $v=$ 1, 2, 3), as plotted in Figs. 4(c) and 4(d), and, correspondingly, 
the spin structure factors have six peaks with equal intensities, as shown in the right two panels of Fig. 5(b). The $n_{\mathrm{sk}}=1 \mathrm{SkX}$ carries a net scalar chirality, which is reduced to around half from the value in the $n_{\mathrm{sk}}=2 \mathrm{SkX}$, as plotted in Fig. 4(b). All these features are similar to the $\operatorname{SkX}$ with $n_{\mathrm{sk}}=1$ found at $A=0$ in the previous study [53]. Note that this $n_{\mathrm{sk}}=1 \mathrm{SkX}$ is similar to those reported in frustrated magnets [18-21], and rather conventional compared to the $n_{\mathrm{sk}}=2$ state.

For a larger magnetic field, the system behaves differently between $A=0.002$ and $A=-0.001$. At $A=0.002$, the $n_{\mathrm{sk}}=$ $1 \mathrm{SkX}$ survives up to $H \sim 0.012$ and turns into another state with a jump of the magnetization and vanishing of the spin scalar chirality, as shown in Figs. 4(a) and 4(b). The highfield state is a topologically trivial $2 Q$ noncoplanar state, as indicated in Figs. 4(c) and 5(c). We note that this state exhibits a $1 Q$ modulation of the spin scalar chirality due to a small peak structure of the $z$-spin component at higher harmonics, as shown in Fig. 5(c). On the other hand, at $A=$ -0.001 , the phase transition from the $n_{\mathrm{sk}}=1 \mathrm{SkX}$ occurs at a much lower field $H \sim 0.005$ to a topologically trivial $3 Q$ noncoplanar state, as shown in Figs. 4(a), 4(b), and 4(d). We present the spin structure in Fig. 5(d); it is characterized by a $2 Q$ modulation in the $x y$ component with equal intensities and a $1 Q$ modulation in the $z$ component. Meanwhile, the chirality pattern is characterized by the $1 Q$ modulation also in this case. With a further increase of $H$, the $z$ component of the magnetization is gradually suppressed and vanishes at $H \sim 0.014$ as shown in Fig. 4(d), which indicates the phase transition to the $2 Q$ noncoplanar state.

Our results indicate that the stability of the $\mathrm{SkX}$ states in the presence of single-ion anisotropy is largely different between the two SkXs. Compared to the result at $A=0$, the field region of the $n_{\mathrm{sk}}=2 \mathrm{SkX}$ does not show a notable change for both $A=0.002$ and $A=-0.001$. On the other hand, the field range of the $n_{\mathrm{sk}}=1 \mathrm{SkX}$ is substantially extended (reduced) for $A=0.002(A=-0.001)$. In the case of easy-plane anisotropy with $A=-0.001$, the $n_{\mathrm{sk}}=1$ state is unstable to the intervening $3 Q$ state that does not appear for the easy-axis case with $A=0.002$. A similar contrasting response to the easy-axis and easy plane anisotropy is found for SkXs in frustrated magnets [19-21].

\section{SUMMARY}

In summary, we have investigated the stability of the SkXs with different topological numbers $\left(n_{\mathrm{sk}}\right)$ in itinerant magnets against single-ion anisotropy and external magnetic field by large-scale numerical simulations based on the KPM-LD method for the Kondo lattice model on a triangular lattice. We showed that the spin structure of the $n_{\mathrm{sk}}=2 \mathrm{SkX}$ is continuously deformed by the anisotropy into an anisotropic $3 Q$ state composed of magnetic vortices in the $x y$-spin component and a sinusoidal wave in the $z$-spin component. Moreover, we found that the system exhibits a topological phase transition from the $n_{\mathrm{sk}}=2 \mathrm{SkX}$ to a $1 Q$ collinear ( $2 Q$ noncoplanar) state while increasing the easy-axis (easy-plane) anisotropy at zero field. We also clarified that the SkXs with $n_{\mathrm{sk}}=2$ and 1 show contrasting behaviors in an applied magnetic field in the presence of single-ion anisotropy; the stable field range of the $n_{\mathrm{sk}}=2 \mathrm{SkX}$ is not much affected by the anisotropy, while the range of the $n_{\mathrm{sk}}=1 \mathrm{SkX}$ is substantially extended (reduced) by the easy-axis (easy-plane) anisotropy. Our result underscores that the unconventional $\mathrm{SkX}$ with $n_{\mathrm{sk}}=2$ is expected to be found in materials with a relatively small spin-charge coupling and small magnetic anisotropy in itinerant magnets. Such conditions may be attained in monolayer metals on substrates [61-66] and in bulk systems where chiral states were recently reported in $\mathrm{SrFeO}_{3}[67,68]$ and $\mathrm{Gd}_{2} \mathrm{PdSi}_{3}$ [69]. More sophisticated analyses, which take account of the
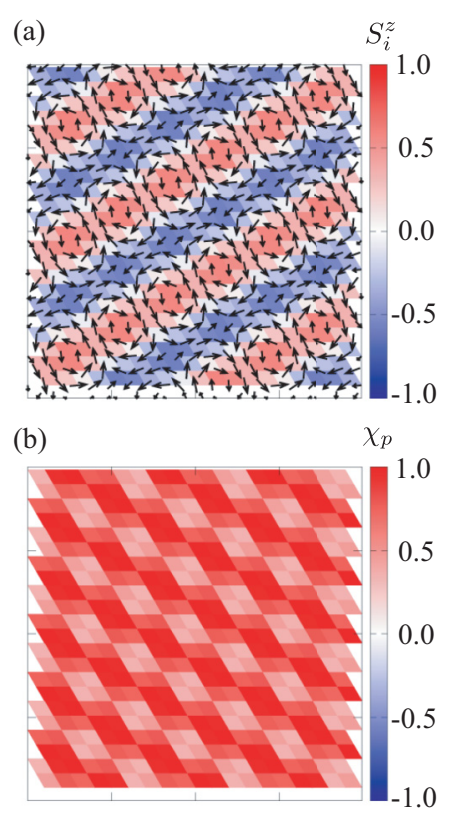
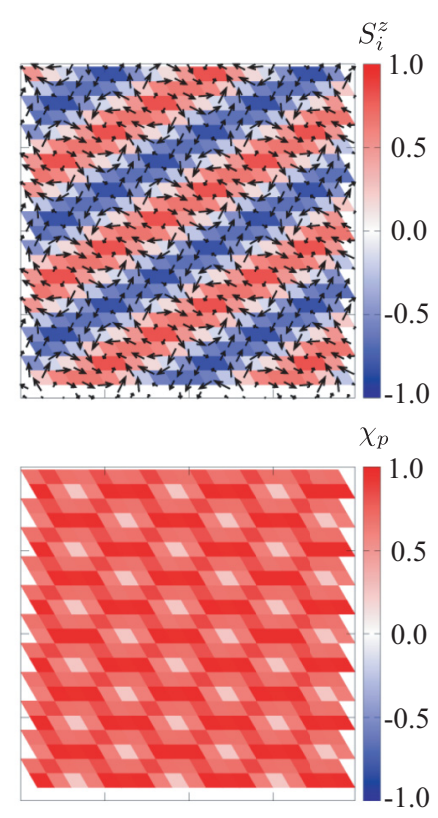
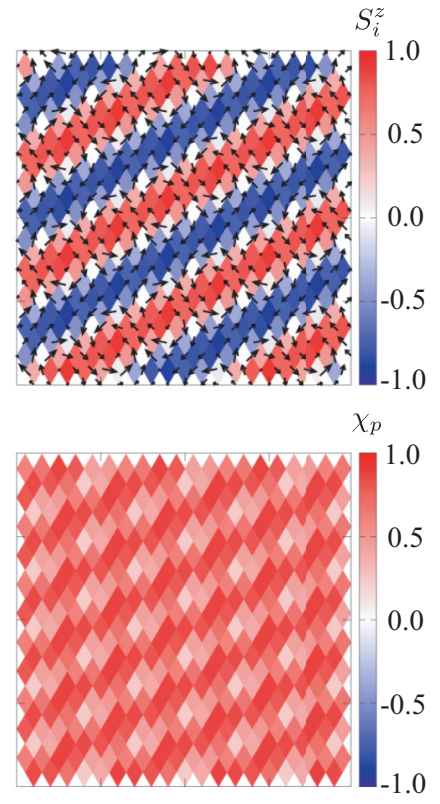
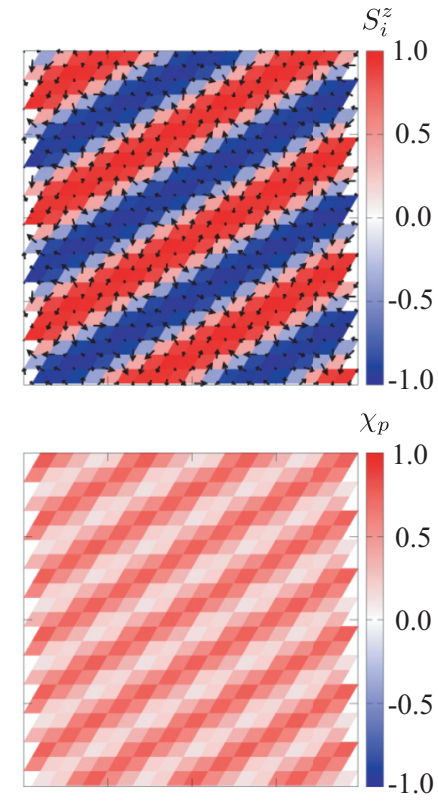

FIG. 6. Snapshots of (a) the spin configurations and (b) the spin scalar chirality in the $3 Q$ SkX with $n_{\mathrm{sk}}=2$. The data are $A=-0.0015$, $0.0015,0.0035$, and 0.0055 from the left panel. 
multiorbital degree of freedom and the spin-orbit coupling, are left for future study.

\section{ACKNOWLEDGMENTS}

The authors acknowledge R. Ozawa and K. Barros for enlightening discussions in the early stage of this study. This research was supported by JSPS KAKENHI Grants No. JP18H04296 (J-Physics) and No. JP18K13488. Parts of the numerical calculations were performed in the supercomputing systems in ISSP, the University of Tokyo.

\section{APPENDIX: REAL-SPACE SPIN CONFIGURATION OF THE $n_{\mathrm{sk}}=2$ SkX FOR DIFFERENT VALUES OF SINGLE-ION ANISOTROPY}

In this Appendix, we show the spin configurations in the $n_{\mathrm{sk}}=2 \mathrm{SkX}$ in the presence of single-ion anisotropy at zero field. Figures 6(a) and 6(b) show the snapshots of the spin configurations and the spin scalar chirality, respectively, obtained by the KPM-LD simulation for $A=-0.0015,0.0015,0.0035$, and 0.0055 .
[1] D. Loss and P. M. Goldbart, Phys. Rev. B 45, 13544 (1992).

[2] J. Ye, Y. B. Kim, A. J. Millis, B. I. Shraiman, P. Majumdar, and Z. Tešanović, Phys. Rev. Lett. 83, 3737 (1999).

[3] K. Ohgushi, S. Murakami, and N. Nagaosa, Phys. Rev. B 62, 6065(R) (2000).

[4] I. Martin and C. D. Batista, Phys. Rev. Lett. 101, 156402 (2008).

[5] Y. Akagi and Y. Motome, J. Phys. Soc. Jpn. 79, 083711 (2010).

[6] R. Shindou and N. Nagaosa, Phys. Rev. Lett. 87, 116801 (2001).

[7] A. N. Bogdanov and D. A. Yablonskii, Sov. Phys. JETP 68, 101 (1989).

[8] A. Bogdanov and A. Hubert, J. Magn. Magn. Mater. 138, 255 (1994).

[9] S. Mühlbauer, B. Binz, F. Jonietz, C. Pfleiderer, A. Rosch, A. Neubauer, R. Georgii, and P. Böni, Science 323, 915 (2009).

[10] X. Yu, Y. Onose, N. Kanazawa, J. Park, J. Han, Y. Matsui, N. Nagaosa, and Y. Tokura, Nature (London) 465, 901 (2010).

[11] X. Z. Yu, N. Kanazawa, Y. Onose, K. Kimoto, W. Z. Zhang, S. Ishiwata, Y. Matsui, and Y. Tokura, Nat. Mater. 10, 106 (2011).

[12] N. Nagaosa and Y. Tokura, Nat. Nanotechnol. 8, 899 (2013).

[13] I. Dzyaloshinsky, J. Phys. Chem. Solids 4, 241 (1958).

[14] T. Moriya, Phys. Rev. 120, 91 (1960).

[15] P. Bak and M. H. Jensen, J. Phys. C: Solid State Phys. 13, L881 (1980).

[16] U. Rößler, A. Bogdanov, and C. Pfleiderer, Nature (London) 442, 797 (2006).

[17] S. D. Yi, S. Onoda, N. Nagaosa, and J. H. Han, Phys. Rev. B 80, 054416 (2009).

[18] T. Okubo, S. Chung, and H. Kawamura, Phys. Rev. Lett. 108, 017206 (2012).

[19] A. O. Leonov and M. Mostovoy, Nat. Commun. 6, 8275 (2015).

[20] S.-Z. Lin and S. Hayami, Phys. Rev. B 93, 064430 (2016).

[21] S. Hayami, S.-Z. Lin, and C. D. Batista, Phys. Rev. B 93, 184413 (2016)

[22] S. Hayami, S.-Z. Lin, Y. Kamiya, and C. D. Batista, Phys. Rev. B 94, 174420 (2016).

[23] P. Sutcliffe, Phys. Rev. Lett. 118, 247203 (2017).

[24] A. B. Butenko, A. A. Leonov, U. K. Rößler, and A. N. Bogdanov, Phys. Rev. B 82, 052403 (2010).

[25] M. N. Wilson, A. B. Butenko, A. N. Bogdanov, and T. L. Monchesky, Phys. Rev. B 89, 094411 (2014).

[26] S.-Z. Lin, A. Saxena, and C. D. Batista, Phys. Rev. B 91, 224407 (2015).

[27] A. Leonov, T. Monchesky, N. Romming, A. Kubetzka, A. Bogdanov, and R. Wiesendanger, New J. Phys. 18, 065003 (2016).
[28] A. O. Leonov and I. Kézsmárki, Phys. Rev. B 96, 014423 (2017).

[29] C. D. Batista, S.-Z. Lin, S. Hayami, and Y. Kamiya, Rep. Prog. Phys. 79, 84504 (2016).

[30] Y. Akagi, M. Udagawa, and Y. Motome, Phys. Rev. Lett. 108, 096401 (2012).

[31] S. Hayami and Y. Motome, Phys. Rev. B 90, 060402 (2014).

[32] R. Ozawa, S. Hayami, K. Barros, G.-W. Chern, Y. Motome, and C. D. Batista, J. Phys. Soc. Jpn. 85, 103703 (2016).

[33] S. Hayami, R. Ozawa, and Y. Motome, Phys. Rev. B 95, 224424 (2017).

[34] S. Hayami, R. Ozawa, and Y. Motome, Phys. Rev. B 94, 024424 (2016).

[35] M. A. Ruderman and C. Kittel, Phys. Rev. 96, 99 (1954).

[36] T. Kasuya, Prog. Theor. Phys. 16, 45 (1956).

[37] K. Yosida, Phys. Rev. 106, 893 (1957).

[38] S. Kumar and J. van den Brink, Phys. Rev. Lett. 105, 216405 (2010).

[39] Y. Kato, I. Martin, and C. D. Batista, Phys. Rev. Lett. 105, 266405 (2010).

[40] S. Hayami and Y. Motome, Phys. Rev. B 91, 075104 (2015).

[41] J. W. F. Venderbos, S. Kourtis, J. van den Brink, and M. Daghofer, Phys. Rev. Lett. 108, 126405 (2012).

[42] J. W. F. Venderbos, Phys. Rev. B 93, 115108 (2016).

[43] S. Sorn, S. Divic, and A. Paramekanti, arXiv:1810.08207.

[44] K. Jiang, Y. Zhang, S. Zhou, and Z. Wang, Phys. Rev. Lett. 114, 216402 (2015).

[45] K. Barros, J. W. F. Venderbos, G.-W. Chern, and C. D. Batista, Phys. Rev. B 90, 245119 (2014).

[46] S. Ghosh, P. O'Brien, C. L. Henley, and M. J. Lawler, Phys. Rev. B 93, 024401 (2016).

[47] S. Hayami and Y. Motome, IEEE Trans. Magn. 55, 1500107 (2018).

[48] S. Hayami and Y. Motome, Phys. Rev. Lett. 121, 137202 (2018).

[49] K. N. Okada, Y. Kato, and Y. Motome, Phys. Rev. B 98, 224406 (2018).

[50] S. Hayami, T. Misawa, Y. Yamaji, and Y. Motome, Phys. Rev. B 89, 085124 (2014).

[51] G.-W. Chern, Phys. Rev. Lett. 105, 226403 (2010).

[52] M. Shahzad and P. Sengupta, Phys. Rev. B 96, 224402 (2017).

[53] R. Ozawa, S. Hayami, and Y. Motome, Phys. Rev. Lett. 118, 147205 (2017).

[54] K. Barros and Y. Kato, Phys. Rev. B 88, 235101 (2013). 
[55] A. Weiße, G. Wellein, A. Alvermann, and H. Fehske, Rev. Mod. Phys. 78, 275 (2006).

[56] Z. Wang, K. Barros, G.-W. Chern, D. L. Maslov, and C. D. Batista, Phys. Rev. Lett. 117, 206601 (2016).

[57] R. Ozawa, S. Hayami, K. Barros, and Y. Motome, Phys. Rev. B 96, 094417 (2017).

[58] G.-W. Chern, K. Barros, Z. Wang, H. Suwa, and C. D. Batista, Phys. Rev. B 97, 035120 (2018).

[59] J. M. Tang and Y. Saad, Numer. Linear Algebra Appl. 19, 485 (2012).

[60] J. H. Mentink, M. V. Tretyakov, A. Fasolino, M. I. Katsnelson, and T. Rasing, J. Phys.: Condens. Matter 22, 176001 (2010).

[61] K. von Bergmann, S. Heinze, M. Bode, E. Y. Vedmedenko, G. Bihlmayer, S. Blügel, and R. Wiesendanger, Phys. Rev. Lett. 96, 167203 (2006).

[62] S. Heinze, K. von Bergmann, M. Menzel, J. Brede, A. Kubetzka, R. Wiesendanger, G. Bihlmayer, and S. Blügel, Nat. Phys. 7, 713 (2011).
[63] Y. Yoshida, S. Schröder, P. Ferriani, D. Serrate, A. Kubetzka, K. von Bergmann, S. Heinze, and R. Wiesendanger, Phys. Rev. Lett. 108, 087205 (2012).

[64] B. Zimmermann, M. Heide, G. Bihlmayer, and S. Blügel, Phys. Rev. B 90, 115427 (2014).

[65] D. Serrate, Y. Yoshida, M. Moro-Lagares, A. Kubetzka, and R. Wiesendanger, Phys. Rev. B 93, 125424 (2016).

[66] M. Hoffmann, B. Zimmermann, G. P. Müller, D. Schürhoff, N. S. Kiselev, C. Melcher, and S. Blügel, Nat. Commun. 8, 308 (2017).

[67] S. Ishiwata, M. Tokunaga, Y. Kaneko, D. Okuyama, Y. Tokunaga, S. Wakimoto, K. Kakurai, T. Arima, Y. Taguchi, and Y. Tokura, Phys. Rev. B 84, 054427 (2011).

[68] S. Ishiwata, T. Nakajima, J.-H. Kim, D. Inosov, N. Kanazawa, J. White, J. Gavilano, R. Georgii, K. Seemann, G. Brandl et al., arXiv:1806.02309.

[69] T. Kurumaji, T. Nakajima, M. Hirschberger, A. Kikkawa, Y. Yamasaki, H. Sagayama, H. Nakao, Y. Taguchi, T.-h. Arima, and Y. Tokura, arXiv:1805.10719. 